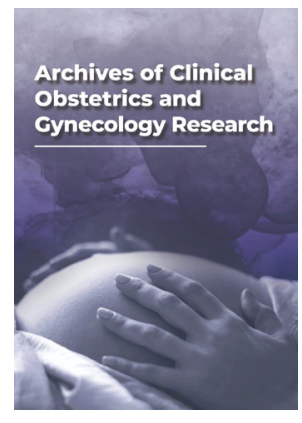

Correspondence

Liu Guolin

The Third People's Hospital of Bengbu City, Anhui Province, PR China

E-mail: liuguolin2013090@163.com

- Received Date: 25 Nov 2020

- Accepted Date: 30 Nov 2020

- Publication Date: 17 Dec 2020

Copyright

(c) 2021 Science Excel. This is an openaccess article distributed under the terms of the Creative Commons Attribution 4.0 International license.

\title{
A case of severe soft birth canal laceration after cervical ligation
}

\author{
Liu Zhijun' Liu Guolin ${ }^{1,2^{*}}$ \\ 'The Third People's Hospital of Bengbu City, Anhui Province, PR China \\ ${ }^{2}$ Anhui Fuyang People's Hospital 236000, Anhui Province, PR China
}

\begin{abstract}
Cervical ligation in early pregnancy is the first choice for the treatment of cervical dysfunction. It can significantly prolong gestational weeks, improve the success rate of pregnancy, and improve the outcome of mother and child. And the incidence of complications of this operation is low, serious complications are very rare, the most common complications include premature rupture of membranes, chorioamnionitis, endometritis, perioperative bleeding, cervical laceration and so on, rare complications include bladder cervical fistula, ureteral cervical fistula and so on. This paper reports a case of severe soft birth canal laceration after cervical ligation, and reviews the relevant literature, summarizes the relevant experience, and provides some suggestions for the diagnosis and treatment of this kind of pregnant women.
\end{abstract}

\section{Clinical information}

Li Yue, Family instead of narration: second child, After 37 weeks of pregnancy, Paroxysmal lower abdominal pain 2 hours into our hospital ". The patient is mentally retarded from childhood, can't live alone, Regular menstruation, The last menstruation is unknown. Family member v. According to early pregnancy ultrasound, the last menstruation is February 30,2020, There was no obvious abnormality in early pregnancy, About 20 weeks after menopause, Establishment of birth check cards in local communities at 12 weeks of gestation, But irregular birth tests during pregnancy, The whole pregnancy prenatal examination was 3 times. Current pregnancy Four weeks, Paroxysmal lower abdominal pain, For 30 to 60 seconds, Between two and three minutes, With vaginal fluid, Clear color, About $300 \mathrm{ml}$, Vaginal examination suggests gluteal exposure, The palace has been opened. In the last 3 days, No bath history, In our hospital, Emergency plan "1. G6P1 pregnancy Four weeks, 2. buttock, 3. mental retardation "income my department. The general condition of pregnancy is fine, Diet, sleep, Normal defecation, There was no significant loss of weight in the near future. Past history: denial of heart, Liver, brain, Kidney, diabetes, History of hypertension, The patient is mentally retarded, The answer is not relevant. Denial of blood transfusions, Allergies, Surgery, Smoking, A history of drinking, etc. History of pregnancy and childbirth :1-0-4-1(her daughter's intelligence is normal). After admission: T: $36.5^{\circ}, \mathrm{P}: 90$ beats/min, R: 20 times per minute, $\mathrm{mmHg}$; $\mathrm{BP}$ : $136 / 87$ There was no obvious abnormality in the remaining related physical examination. Obstetric examination $: 30 \mathrm{~cm}$, high Abdominal cm, 95 LSA, of birth orientation Fetal heart 140 beats/min, The contractionslasted 30 to 60 seconds, Between two and three minutes, Show your hips, S 3, The membranes are broken, Amniotic fluid, Open your mouth, Estimated fetal weight about $2800 \pm 200 \mathrm{~g}$. Auxiliary examination: ultrasound and other examinations are not available. After admission, the emergency department improved the relevant hematological examination, informed the family of the current situation of pregnant women, suggested vaginal delivery, the family expressed understanding. After 8 minutes, hip traction assisted delivery of a baby girl, weight $2800 \mathrm{~g}$, the delivery process is smooth. After placenta stripping, vaginal bleeding is less. Routine examination of soft birth canal, exploration of cervical mouth integrity, did not touch obvious laceration, in the external mouth of the cervix touch a similar birth control ring object, consider with device pregnancy, touch the ring back outward pull, pull failure. After using vaginal retractor to open vagina, silk thread and fine rubber strip were fixed on cervix, and the family history was asked again to the pregnant woman's family. The family carefully recalled that pregnant women in other hospitals about 20 weeks due to cervical dysfunction, cervical ligation, and no follow-up. When the cervical suture was removed, vaginal bleeding began to increase, and the soft birth canal was carefully examined again. A laceration was seen about $3 \mathrm{~cm}$ above the cervix, extending upward, about 6 to $7 \mathrm{~cm}$, deep into the posterior fornix, the posterior fornix was intact, the uterine contractility was normal after routine suture with absorbable suture No .2, the vaginal discharge was still seen in the vagina, the vagina was opened again, and the suture continued observation showed that there was still a small stream-like bright red blood outflow, considering that the soft birth canal laceration reached the posterior fornix, the possibility of incomplete rupture of the uterus 
was not ruled out, and further difficulty in exploring the vagina was considered, And the suture is difficult, inform the pregnant woman's family situation, suggest to carry on the laparotomy exploration, after the doctor-patient communication carries on the laparotomy exploration operation, at this time the total amount of vaginal bleeding is estimated to be about $800 \mathrm{ml}$, to prepare the blood transfusion, parallel emergency laparotomy exploration.Preoperative diagnosis :1. Soft birth canal laceration 2. Postpartum hemorrhage 3. Pregnancy with cervical ligation 4. Cervical insufficiency 5. Hip position 6. G6P2 pregnancy 374 weeks 7. Mental retardation. To explore the uterus, Lower uterine segment, Bilateral appendages, There was no abnormality in the appearance of the retroperitoneal and posterior domes of the bladder, At this point the contractions are poor, A 250- ug. injection The posterior vault was full, About $6 \mathrm{~cm}$, diameter From the abdominal compression of the posterior fornix, About $300 \mathrm{ml}$ of blood clots came out of the vagina, The fullness of the posterior vault disappears, At the same time, there is active bleeding between the vagina and the posterior vault, Vaginal suture is difficult, Under vaginal instructions, Transabdominal No .0 absorbable suture at the posterior fornix under the cervix continuous spiral suture. Re-exploration of the vagina without significant bleeding, Suture the perineal incision, Sterile gauze 2 fast compression of vaginal wall. To explore the abdominal cavity again, The palace, Lower uterine segment, Bilateral appendages, No hematoma at the retroreflective peritoneum and posterior vault, No significant bleeding at the suture, Ornidazole to flush the abdominal cavity, Close your belly, About 1,500 ml, of bleeding To correct anemia and symptomatic treatment, Six days after surgery, Good recovery..

\section{Discussion}

UCervical dysfunction refers to pregnancy, At the time of full term pregnancy, the cervical canal was flattened, thinned, dilated and widened, Which eventually leads to an abortion, Cervical dysfunction is the most common cause of habitual abortion in mid-term pregnancy. Related literature reports that $20 \%$ to $25 \%$ of mid-pregnancy abortion is due to cervical dysfunction. Cervical dysfunction is a clinical diagnosis, The diagnosis is vague, Lack of objective gold standards. Most scholars believe that cervical laceration induced by labor and induced labor, cervical conization, cervical ring resection, congenital mallet tube dysplasia, lack of cervical collagen and elastin, exposureene female phenol exposure and cervical tissue structure defects are the high risk factors. The main diagnostic basis $: 1,1$ or 2 times during the same gestational week, And no obvious abdominal pain or contractions, (a) Abortion that is progressing rapidly during the birth process; During pregnancy, the length of the cervical canal was found to last less than $25 \mathrm{~mm}$; 2 . others such as uterine fallopian tube shadowing, Cervical balloon traction camera, During luteal phase, cervical dilatation was evaluated by cervical dilator 7 or $8[1]$. The main treatment methods are nonsurgical treatment and surgical treatment. Most researchers believe that non-surgical treatments such as restricted mobility, bed rest, and pelvic support devices are not effective. So far, Cervical ligation is still the only effective [2] for the treatment of cervical dysfunction. Most scholars believe that cervical ligation strengthens the tension of the cervical canal to the greatest extent possible, And prevent the extension of the lower uterine segment and cervical dilatation; Increased the cervical intratumoral bearing late pregnancy fetal and fetal appendages gravity; Meanwhile, After surgery, fetal preservation may reduce the tension of uterine muscle and lower uterine load; Cervical ligation weakens the defects of the cervix itself, Maintain cervical length and retain cervical mucus suppository.

There are corresponding complications in any operation. Although the operation of cervical ligation is simple, after decades of development, its technology has been continuously improved, making the incidence of complications low, and serious complications are very rare. At present, the most common complications are premature rupture of membranes, chorioamnionitis, endometritis, perioperative bleeding, cervical laceration, circumferential or circumferential band displacement, etc. The rare complications include bladder cervical fistula, ureteral cervical fistula and so on.
Uterine rupture, septicemia is extremely rare, but there is still a possibility of [3]. Either complication is fatal to pregnant women and may cause adverse maternal and infant pregnancy outcomes.

The incidence of various complications varies with the timing, indication, operation and timing of cervical ligation. In this case, cervical ligation causes severe soft birth canal laceration and postpartum hemorrhage, and requires laparotomy to repair soft birth canal laceration. Looking back on the pregnancy management process of the patient, the whole pregnant woman did not carry out a standardized prenatal examination throughout the pregnancy, and the pregnant woman and the family of the pregnant woman could not provide the corresponding medical history after hospitalization. After placental dissection, the routine exploration of soft birth canal found that the cervix was intact and not torn, but the cervical orifice touched a circular object similar to the birth control ring, and only after the failure was taken out was the silk thread and rubber strip of cervical ligation. Ask the patient's family history and provide a history of surgery. At this time we realize the seriousness of the problem, the cervix after cervical ligation is complete, then the fetus is from which part of the soft birth canal delivery. After the vaginal retractor opened the vagina, the soft birth canal laceration was found, the posterior cervix was torn upward, the posterior fornix was deep, postpartum hemorrhage occurred after vaginal suture, and the suture of soft birth canal laceration was performed after vaginal suture failed. Smooth repair of soft birth canal laceration, avoid hysterectomy. As far as the patient is concerned, even if we know before the birth of the cervical ring ligation, as far as the situation is concerned, the opening of the uterine mouth, first exposed buttocks, $\mathrm{S} 3$, we cannot remove the cervical ring line in time to avoid soft birth canal laceration.

Traditional McDonald and Shirodkar surgery is performed through the vagina, using non-absorbable thick silk thread or nylon thread suture, and the fetus can be delivered through the vagina after removing the suture. Abdominal cervical isthmus ligation can use polypropylene ring ligation, generally do not remove [4]. For pregnant women without complications and planning vaginal delivery of McDonald surgery, it is generally recommended to remove the ligation line at 36-37 weeks of pregnancy. Here we should understand that removing the pointer of the ligation line is not delivery, so as not to cause serious consequences. For elective cesarean section, it is recommended to remove sutures at the same time. Pregnant women after transabdominal cervical isthmus ligation suggest elective cesarean section to terminate pregnancy, generally do not remove the ring ligation line. If obstetric complications require early termination of pregnancy, if vaginal delivery is allowed, the circumferential ligation line can be removed. If not, cesarean section terminates the pregnancy [5]. The opinion of immediate removal $(<24 \mathrm{~h})$ or delayed removal $(>24 \mathrm{~h}$ ) is not uniform for patients with premature rupture of membranes. It is necessary to consider the timing [6] of suture removal combined with gestational weeks and possible complications. For patients with premature birth symptoms after ligation, the removal of sutures should be careful, and those with threatened premature birth symptoms should be treated with routine treatment, such as exacerbation of symptoms, changes in cervical canal dilatation, and regular uterine contraction. Remove the [7] of ligation when vaginal bleeding increases. As far as this patient is concerned, the main cause of complications is the serious consequences of improper removal of cervical ligation, warning us to pay attention to the timing of removal of ligation in such patients.

Cervical ligation is the most effective method to treat cervical dysfunction. Although its complications are few, the consequences of various complications are serious. We should pay attention to the monitoring and management of pregnancy after ligation: according to the management of high-risk pregnancy; the operator should pay attention to the matters needing attention, preferably follow up this kind of pregnant women regularly; pay attention to the length of cervix and the shape of cervix; pay attention to the screening and treatment of vaginal infection. 


\section{References}

1. ELan S. Interpretation of guidelines for the treatment of cervical ysfunction ACOG cervical cercular ligation. International Journal of Obstetrics and Gynecology. 2016;43(6):652-653.

2. American College of Obstetricians and Gynecologists. ACOG Practice Bulletin No.142: Cerclage for the management of cervical insufficiency. Obstet Gynecol, 2014;123(2 Pt 1)372-379.

3. Burger NB, Brölmann HA, Einarsson JL, et al. Effectiveness of abdominal cerclage placed via laparotomy or laparoscopy: systematic review. J Minim Invasive Gynecol. 2011;18(6):696704.

4. Xue L, Hong Z. Cervical insufficiency and preterm birth. Chinese Journal of Practical Gynecology and Obstetrics. 2018;34(2):147-148.
5. Sundtoft I, Langhoff-Roos J, Sandager P, et al. Cervical collagen is reduced in non-pregnant women with a history of cervical in-sufficiency and a short cervix. Acta Obstet Gynecol Scand. 2017;96(8)984-990.

6. De Laskin M, Yinon Y, Whittle WL. Preterm premature rupture of membranes in the presence of cerclage is the risk for intrauterine infection and adverse neonatal outcome increased? J Matern Fetal Neonatal Med. 2012;25(4)424-428.

7. Perlman J. Periviable birth: executive summary of a joint workshop by the Eunice Kennedy Shriver National Institute of Child Health and Human Development, Society for Maternal -Fetal Medicine,American Academy of Pediatrics, and American College of Obstetricians and Gynecologists. Obstet Gynecol. 2014;124(3)635-636. 